\title{
Mechatronics system design and experiment research for a novel patient transfer apparatus
}

\author{
Hongbo Wang ${ }^{1, a^{*}}$, Liyu Xie ${ }^{1, b}$, Xiong Zhao ${ }^{1, c}$, Jianye Niu ${ }^{1, d}$, \\ Zhengcao Liu, ${ }^{1, \mathrm{e}}$, Luige Vladareanu ${ }^{2, \mathrm{f}}$, Razvan-Viorel Mihai ${ }^{2, \mathrm{~g}}$ \\ ${ }^{1}$ Parallel Robot and Mechatronic System Laboratory of Hebei Province, Yanshan University, \\ Qinhuangdao 066004, China \\ ${ }^{2}$ Key Laboratory of Mechatronics, Department of Mechatronics, Insitute of Solid Mechanics - \\ Romanian Academy of Bucharest, Romania

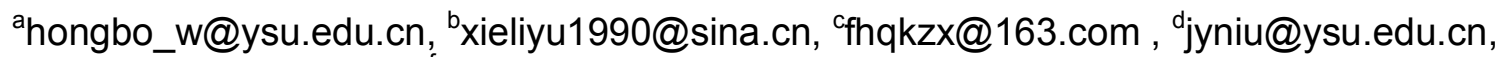 \\ egfxmysu@gmail.com, luigiv2007@yahoo.com.sg, ${ }^{9}$ razvan.viorel_mihai@yahoo.com
}

\begin{abstract}
Keywords: patient transfer apparatus, mechanism design, control system design, transfer experiment.

Abstract. A novel patient transfer apparatus is presented in this paper. First of all, according to the hospital practical requirements, the design demands are analyzed. Secondly, based on the design demands and modular theory, the design idea of the apparatus consisting of four upper sub-modules and four lower sub-modules, all of them being commanded by four controllers, is proposed. All the modules are driven by belts. Next, the mechanism design and electronic control system design are described. The control system is composed of the master computer and the slave computer, which can realize the current control mode, speed control mode and position control mode. Finally, the actual transfer experiment is conducted to verify the effectiveness of the patient transfer apparatus.
\end{abstract}

\section{Introduction}

Currently, the transfer of patients in hospitals is still based on the traditional methods that nurses lift and hold the patients onto stretchers. It brings nurses high labor intensity and may aggravate the patients' injury[1]. Fig. 1 shows a kind of apparatus which can transfer patients with their lying postures unchanged [2]. The special sheet under the patient is slung to the outrigger. Due to the lifting sheet being soft, it exists a certain risk to worsen patients' situation. The Tokyo University develops a kind of nursing robot, as is shown in Fig. 2 [3]. The robot has double arms which could imitate human's arms to insert under a patient's body and transfer him. However, patients have to change their postures in the transfer process. So the robot cannot be applied to critical patients. Fig. 3 shows a LW-TB-1 transfer apparatus developed by Dalian Longwei medical company. This apparatus can accurately and automatically transfer patients. The fact that the apparatus plate unit cannot be divided from the walking unit makes the apparatus bulky and limited in working conditions.

For the above problems, this paper presents a novel patient transfer apparatus that is based on the modular theory, as shown in Fig. 4. The transfer apparatus's size is small and it can be operated conveniently. It needn't to change patients' postures, thus the patient's condition won't aggravate.

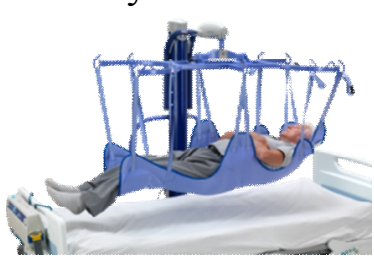

Fig. 1 Sling transfer

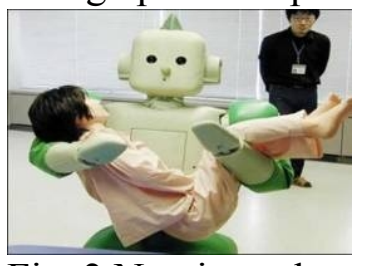

Fig. 2 Nursing robot

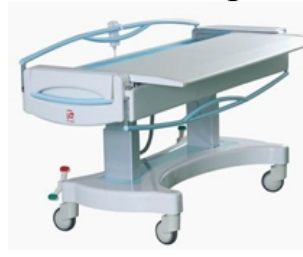

Fig.3 LW-TB-1

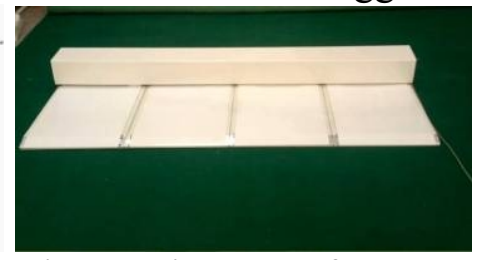

Fig.4 Patient transfer apparutus

\section{Design demands.}

To ensure painless transfer under patients' lying postures, the patient transfer apparatus needs to meet the following demands when designed: 
(1) The apparatus rotation, patient rotation function, as well as lifting, carrying and unloading capability need to be implemented in order to realize a proper transfer.

(2) The apparatus is operated using wireless commands. The operation modes are easy and simple to set. Thus, it only needs one person to complete it.

(3) On the bottom of the apparatus and the front of the cover, proximity sensors are mounted. When a falling tendency appears or the apparatus extrudes patients, an alarm will be activated.

\section{Principle of operation.}

The patient transfer apparatus runs by driving the belts surrounding the upper and the lower sub-modules, as is shown in Fig. 5. Six operation modes are possible:

(1) Empty running: the upper belts don't rotate, while the lower belts rotate counterclockwise.

(2) Patients lifting: the upper belts rotate clockwise and the lower belts rotate counterclockwise.

(3) Manned running: the upper belts don't rotate and the lower belts rotate clockwise.

(4) Patients unloading: the upper belts rotate counterclockwise and the lower belts rotate clockwise.

(5) Apparatus rotating: the lower belts which are corresponding to head and chest rotate opposite to those which are corresponding to haunch and legs.

(6) Patients rotating: the upper belts which are corresponding to head and chest rotate opposite to those which are corresponding to haunch and legs.

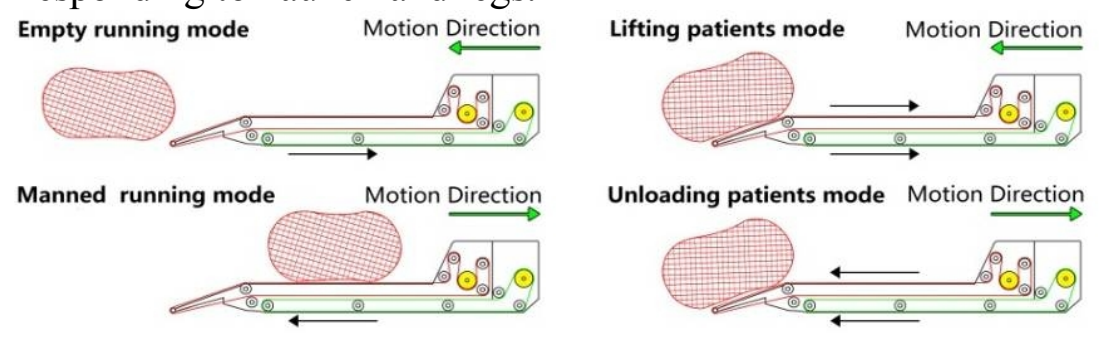

Fig.5 Operation principles

\section{Mechanism design}

Corresponding to the following four parts of human body (head, chest, haunch and legs), the apparatus is divided into four modules, and each module consists of two independent sub-modules. The upper sub-modules realize the function of adjusting patient's location, while the lower sub-modules realize the function of adjusting the apparatus's location. Therefore, the different modules speed has no influence on patients. In order to decrease the thickness of the apparatus so that it can improve the comfort degree of patients, control system is placed in the lateral side of the apparatus. The four upper and lower sub-modules are positioned in the middle of the frame plate.

The upper sub-module. The upper sub-module consists of four parts as drive motor unit, the rollers unit, the belts and the frame. It lifts, unloads and rotates the patient's body by the static friction between skin surface and the belt. The transmission belt system of upper sub-module is divided into three sections: driving section, horizontal section and inclined section. As shown in Fig. 6, the roller 1 is the driving roller. In order to increase the static friction between the driving roller and the belt, some rubber is coated over the driving roller. Roller 2 and roller 3 can increase the wrap angle of the driving roller, and can also tight the belt by adjusting the sliders in the end. The tightening can prevent the belts from slacking and slipping after a long time usage. Roller 6 composes the inclined section together with roller 7 . To decrease the thickness of the upper sub-modules, roller 8 is added. The inclined section of the upper sub-module, namely the end insertion of the apparatus, is designed as a smooth transition wedge. When the apparatus runs, the belt can be worn out badly because of the bending of the guide rollers at the end insertion. Once the deflection is big enough, the spindle head of rollers can escape from the bearings. To decrease the span of guide rollers and increase its flexural rigidity, two support bases are set in the middle of the end insertion to support the guide rollers. 
The lower sub-module. The lower sub-module is composed of the motor driving unit, synchronous pulley unit, the frame and a synchronous belt. The main function of this module is to complete the movement of the apparatus. Nine synchronous pulleys are arranged along the frame. Therefore, the adhesion and the load capacity can be enhanced in order to achieve a stable running operation. Due to the plastic deformation, the synchronous belt will stretch after running a long time. So a tension device is mounted on the lower sub-module frame, which can ensure the synchronous belt tension by changing the distance of the two half-frames. The structure of the lower sub-module is shown in Fig.7.

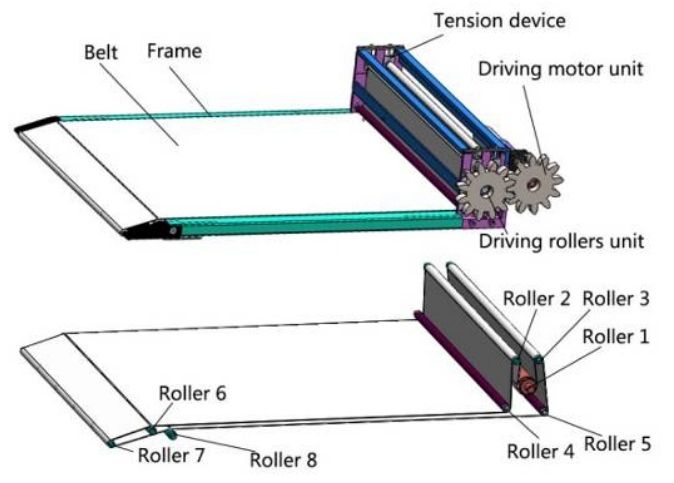

Fig. 6 Upper sub-module

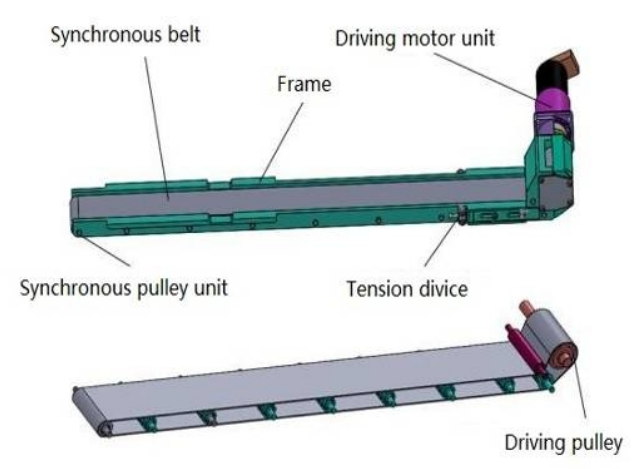

Fig. 7 Lower sub-module

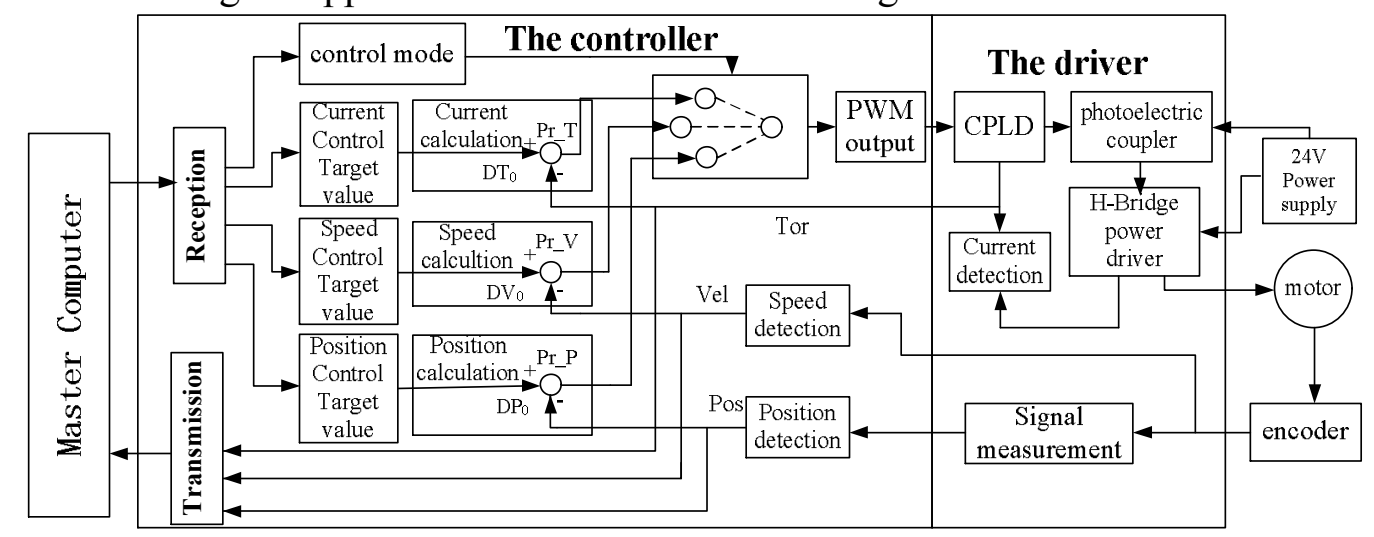

Fig. 8 Control block diagram

\section{Electronic control system}

The apparatus control system is composed of a master computer and a slave computer. An arm S3C2440 represents the master computer, while a group of 4 electronic modules represent the slave computer (each module consists of one controller and 2 motor divers, one for the upper sub-module and another for the lower sub-module). The operation command is sent by the operation box and received by the master computer. And the master computer sends the desired mode operation to slave computer (the controller and the two drivers). The communication protocol between the master computer and the slave computer is relaying on CAN communication protocol by sending commands to the slave computer and collecting data from it. The proximity sensors group of sends the collected data directly to the master computer. The controller and the driver are developed independently. Current, speed and position control modes can be realized. Based on the controller's PWM, the driver controls the motor through a signal and sent the result to the controller to adjust duty ratio of PWM based on the PI arithmetic. The incremental photoelectric encoder is mounted on the shaft of each motor, and it sends pulse signals to the controller in order to calculate the speed and the position of the motor. Then, a three closed-loop servo control system is implemented on the controller, thus the mechanical origin through an origin position detection circuit can be found. Fig. 8 shows the control block diagram.

When transferring, the upper computer detects the position of each motor of lower sub-modules to avoid deviating from straight path. At the bottom of the apparatus, 4 proximity sensors to guard 
apparatus's falling are mounted. At the front side of the cover, there is a proximity sensor, which can detect the position of the patient and avoid extruding patients.

\section{Transfer experiment}

To verify if the patient transfer apparatus can complete the specific movements, a human experiment is carried out. General transfer process is shown in Fig. 9, which includes lifting, carrying, and unloading the patient. Fig. 10 show that when the apparatus is inclined, it can return to the right location by controlling the four motors of the lower sub-modules. The experiment demonstrated that patient transfer apparatus can complete the required movements to transfer the patients without changing the posture.

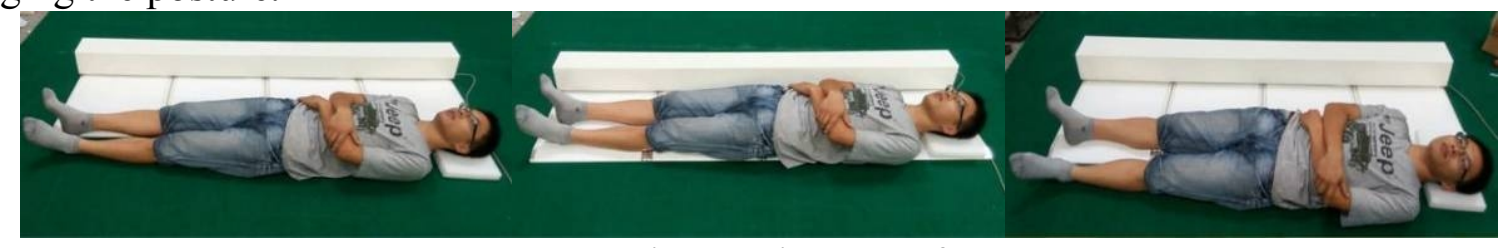

Fig.9 Patient transfer

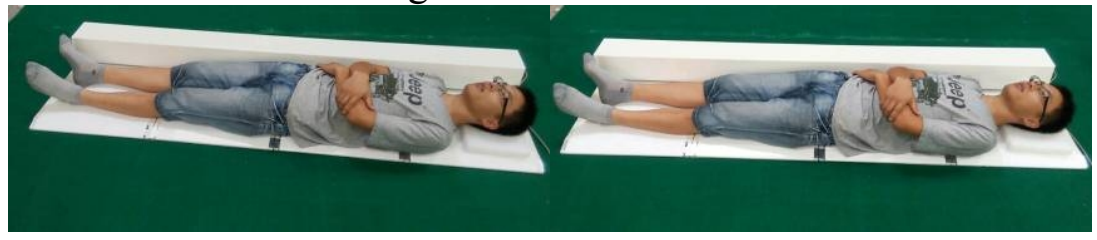

Fig. 10 Apparatus rotation

\section{Conclusion}

The novel patient transfer apparatus presented in this paper can realize comfortable and painless transfer. In addition, it needn't to change patients' postures, so it can be used to transfer fracture patients and patients before and after operation. Therefore, it can ease labor intensity of nurses and improve the medical service level.

\section{Acknowledgment}

This work was supported by National Science and Technology Support Program (2015BAI06B01) and The Hundred Talents Program of Hebei Province.

\section{References}

[1] Evanoff B., Wolf L., Aton E., et al. Reduction in Injury Rates in Nursing Personnel through Introduction of Mechanical Lifts in The Workplace. American Journal of Industrial Medicine, 2003, 44(5): 451-457.

[2] Simon W. H. Storable Patient Lift and Transfer Apparatus. US: 5560054, 1996-10-1.

[3] Onishi M., Luo Z. W., Odashima T., et al. Generation of Human Care Behaviors by Human-Interactive Robot RI-MAN//Proceedings of IEEE International Conference on Networking, Robotics and Automation, Roma, 2007: 3128-3129.

[4] Bhat R., Van E. A. Trolley for Transporting a Patient and a Patient Handling System. US: 12092425, 2006-10-24.

[5] Humphreys H. C., Book W J. Hydraulically Actuated Patient Transfer Device with Passivity based Control. Sarasota, FL, United states: American Society of Mechanical Engineers, 2013.

[6] Huang Z F., Nagata A., Kanai-Pak M., et al. Design of a Robot for Patient Transfer Training. Nagoya, Japan: Society of Instrument and Control Engineers (SICE), 2013: 1041-1046. 